\title{
New integral formulae for two complementary orthogonal distributions on Riemannian manifolds
}

\author{
Magdalena Lużyńczyk ${ }^{1}$ • Paweł Walczak ${ }^{1}$
}

Received: 28 January 2015 / Accepted: 19 April 2015 / Published online: 19 May 2015

(C) The Author(s) 2015. This article is published with open access at Springerlink.com

\begin{abstract}
We derive and apply a new integral formula for a closed Riemannian manifold equipped with a pair of complementary orthogonal distributions (plane fields). The integrand depends on the second fundamental forms and integrability tensors of the distributions, their covariant derivatives, and of the Ricci curvature of the ambient manifold. Also, we discuss some applications of this formula and of another formula of this sort, the one obtained earlier by the second author, and show that both formulae may hold when the distributions are defined only outside a "reasonable" closed subset of the manifold under consideration.
\end{abstract}

Keywords Riemannian manifold · Distribution · Foliation · Integral formula

Mathematics Subject Classification Primary 53C15 - Secondary 53C12

\section{Introduction}

Integral formulae for foliated Riemannian manifold are almost that old as the foliation theory itself: already in 1950, Georges Reeb [12] has shown that the integral of the mean curvature of the leaves of a codimension-one foliation of a closed oriented Riemannian manifold is always equal to zero. Later on, Asimov [4] and Brito et al. [6] have shown that integrals of mean curvatures (of arbitrary higher order $k$ ) of codimension-one foliations $\mathcal{F}$ of closed manifolds $M$ of constant curvature $c$ depend only on $k, c$, volume and dimension of $M$, not on $\mathcal{F}$. Next, one of this formulae has been extended to foliations of arbitrary Riemannian manifolds:

\footnotetext{
$凶$ Paweł Walczak

pawelwal@math.uni.lodz.pl

Magdalena Lużyńczyk luzynczyk@math.uni.lodz.pl

1 Wydział Matematyki i Informatyki, Uniwersytet Łódzki, Łódź, Poland
} 


$$
\int_{M}\left(2 \sigma_{2}-\operatorname{Ric}(N)\right) \mathrm{d} \operatorname{vol}=0
$$

where $\sigma_{2}$ is the second mean curvature of the leaves of $\mathcal{F}$ and $N$ is a unit vector field orthogonal to $M$. In [19] (see also [11]) formula (1) has been generalized to foliations (and arbitrary distributions) of arbitrary codimension: the result of this generalization can be found here [Sect. 2, Eq. (4)] and has been applied by several authors in different contexts (see [5,7,14,17,18] etc.). Recently, first Rovenski and the second author [15] on symmetric spaces and then Andrzejewski [2] (see also [3]) on arbitrary Riemannian manifolds have found series of integral formulae for codimension-one foliations, formulae which extend (1) and all the equalities proved in [4] and [6]. For more about integral formulae for foliations, we refer to [16].

In this article, we consider a Riemannian manifold $M$ equipped with two complementary orthogonal distributions $D_{1}$ and $D_{2}$. We propose a method of obtaining a series of formulae generalizing (4) and derive one of them (Sect. 2, Eq. (25)). We show that under some conditions both equations, (4) and (25), hold when our distributions $D_{1}$ and $D_{2}$ admit singularities, that is are defined on a closed manifold $M$ apart from a finite union $\Sigma$ of closed submanifolds of sufficiently large codimension. This should be of some interest: existence of distributions on a closed manifold $M$ depends on some topological conditions and implies existence of such distribution over open subsets of $M$, while the converse is not true. Finally, we collect some applications of (4) and (25): it occurs that our formulae provide obstructions for the existence of pairs of distributions satisfying particular geometrical conditions.

The main idea is (as in the case of formulae obtained earlier) to find a geometrically interesting vector field, calculate its divergence and use the classical Stokes Theorem. For distributions with singularities, we need a particular technical result (Lemma 1) which generalizes that from [8]. One of the other tools used in derivation of (25) is an analog of the classical Codazzi equation for non-integrable distributions (Proposition 1).

\section{Preliminaries}

Let $D$ be a distribution on a Riemannian manifold $(M,\langle\rangle$,$) . The second fundamental form$ $B$ of $D$ is defined as follows (see [13]). If $X$ and $Y$ are two vector fields tangent to $D$, then $B(X, Y)$ is the orthogonal to $D$ component of

$$
\frac{1}{2}\left(\nabla_{X} Y+\nabla_{Y} X\right)
$$

where $\nabla$ is the Levi-Civita connection on $M$. The trace $H$ of $B$ is called the mean curvature vector of $D$. Similarly, the integrability tensor $T$ of $D$ assigns to $X$ and $Y$, as before two vector fields tangent to $D$, the orthogonal to $D$ component of

$$
\frac{1}{2}\left(\nabla_{X} Y-\nabla_{Y} X\right)
$$

Hereafter, we deal with two orthogonal distributions $D_{1}$ and $D_{2}$ on a Riemannian manifold $(M,\langle\rangle$,$) . We put p=\operatorname{dim} D_{1}, q=\operatorname{dim} D_{2}$ and $m=\operatorname{dim} M$, and assume that $p+q=m$. For any $v \in T M$, we write

$$
v=v^{\top}+v^{\perp}
$$


where $v^{\top} \in D_{1}$ and $v^{\perp} \in D_{2}$. Throughout the article, we shall use a local orthonormal frame $e_{1}, \ldots, e_{m}$ adapted to $D_{1}$ and $D_{2}$, i.e., we assume that $e_{i}$ is tangent to $D_{1}$ for $i=1, \ldots, p$ and $e_{\alpha}$ is tangent to $D_{2}$ for $\alpha=p+1, \ldots, m$.

With this notation, the second fundamental forms $B_{i}$ of $D_{i}(i=1,2)$ are defined as follows:

$$
B_{1}\left(X_{1}, Y_{1}\right)=\frac{1}{2}\left(\nabla_{X_{1}} Y_{1}+\nabla_{Y_{1}} X_{1}\right)^{\perp}, \quad B_{2}\left(X_{2}, Y_{2}\right)=\frac{1}{2}\left(\nabla_{X_{2}} Y_{2}+\nabla_{Y_{2}} X_{2}\right)^{\top}
$$

for vector fields $X_{i}$ and $Y_{i}$ tangent to $D_{i}$.

Similarly, the integrability tensors $T_{i}$ of $D_{i}(i=1,2)$ are defined by

$$
T_{1}\left(X_{1}, Y_{1}\right)=\frac{1}{2}\left[X_{1}, Y_{1}\right]^{\perp}, \quad T_{2}\left(X_{2}, Y_{2}\right)=\frac{1}{2}\left[X_{2}, Y_{2}\right]^{\top}
$$

for vector fields $X_{i}, Y_{i} \in D_{i}$. Therefore, the distribution $D_{i}$ is integrable (and defines a foliation) if and only if $T_{i}=0$.

Then, the mean curvature vectors $H_{i}$ of $D_{i}$ are given by

$$
\begin{aligned}
& H_{1}=\text { Trace } B_{1}=\sum_{i} B_{1}\left(e_{i}, e_{i}\right)=\sum_{i}\left(\nabla_{e_{i}} e_{i}\right)^{\perp}, \\
& H_{2}=\text { Trace } B_{2}=\sum_{\alpha} B_{2}\left(e_{\alpha}, e_{\alpha}\right)=\sum_{i}\left(\nabla_{e_{\alpha}} e_{\alpha}\right)^{\top} .
\end{aligned}
$$

Let us define also the Weingarten operators $A_{1}: D_{1} \times D_{2} \rightarrow D_{1}$ and $A_{2}: D_{2} \times D_{1} \rightarrow D_{2}$ of our distributions $D_{1}$ and $D_{2}$, respectively, by

$$
\left\langle A_{1}(X, N), Y\right\rangle=\left\langle B_{1}(X, Y), N\right\rangle \text { for } X, Y \in D_{1}, N \in D_{2},
$$

and

$$
\left\langle A_{2}\left(X^{\prime}, N^{\prime}\right), Y^{\prime}\right\rangle=\left\langle B_{2}\left(X^{\prime}, Y^{\prime}\right), N^{\prime}\right\rangle \text { for } X^{\prime}, Y^{\prime} \in D_{2}, N^{\prime} \in D_{1} \text {. }
$$

Finally, let us define the transformations

$$
C_{1}=A_{1}\left(\cdot, H_{1}\right): D_{1} \rightarrow D_{1}, \quad \text { and } \quad C_{2}=A_{2}\left(\cdot, H_{2}\right): D_{2} \rightarrow D_{2} .
$$

Since $C_{i}, i=1,2$, are endomorphisms, we can compose

$$
C_{i}^{k}=C_{i} \circ \cdots \circ C_{i}
$$

$k$-times and consider the vector fields

$$
Z_{k}=C_{1}^{k}\left(H_{2}\right)+C_{2}^{k}\left(H_{1}\right) .
$$

Certainly, $Z_{k}, k=0,1,2, \ldots$, are global vector fields on $M$ defined by means of geometry of $M, D_{1}$ and $D_{2}$.

\section{The formulae}

For $k=0$ in (2), we get immediately

$$
Z_{0}=H_{1}+H_{2}
$$


The divergence $\operatorname{div}\left(H_{1}+H_{2}\right)$ was calculated in [19]:

$$
\begin{aligned}
\operatorname{div}\left(H_{1}+H_{2}\right)= & K\left(D_{1}, D_{2}\right)+\left\|B_{1}\right\|^{2}+\left\|B_{2}\right\|^{2} \\
& -\left\|H_{1}\right\|^{2}-\left\|H_{2}\right\|^{2}-\left\|T_{1}\right\|^{2}-\left\|T_{2}\right\|^{2},
\end{aligned}
$$

where $K\left(D_{1}, D_{2}\right)$ is a generalization of the Ricci curvature given by

$$
K\left(D_{1}, D_{2}\right)=\sum_{i, \alpha}\left\langle R\left(e_{i}, e_{\alpha}\right) e_{\alpha}, e_{i}\right\rangle
$$

and called the mixed scalar curvature. If $M$ is closed and oriented, integrating both sides of (3) and using the Stokes Theorem, we get the integral formula

$$
\begin{aligned}
& \int_{M}\left(K\left(D_{1}, D_{2}\right)+\left\|B_{1}\right\|^{2}+\left\|B_{2}\right\|^{2}\right. \\
& \left.\quad-\left\|H_{1}\right\|^{2}-\left\|H_{2}\right\|^{2}-\left\|T_{1}\right\|^{2}-\left\|T_{2}\right\|^{2}\right) \mathrm{d} \text { vol }=0 .
\end{aligned}
$$

Now, we shall take $k=1$ in (2). As we shall see, the calculation and the resulting integral formula (25) below, are rather complicated, but still applicable. Certainly, one can work also with the fields $Z_{k}$ for $k>1$ to get integral formulae of the form

$$
\int_{M} \operatorname{div} Z_{k} \mathrm{~d} \text { vol }=0
$$

with div $Z_{k}$ expressed in terms of $B_{i}, T_{i}, H_{i}, R$ and their covariant derivatives, but it seems to us that the formulae obtained in this way will be even more complicated and less interesting than (25), so we decided not to proceed this way, but to concentrate on collecting applications of (4) and (25).

First, consecutive calculations yield the following:

$$
\begin{aligned}
\operatorname{div} Z_{1}= & \operatorname{div}\left(A_{1}\left(H_{2}, H_{1}\right)+A_{2}\left(H_{1}, H_{2}\right)\right) \\
= & \sum_{i}\left\langle\nabla_{e_{i}}\left(A_{1}\left(H_{2}, H_{1}\right)\right), e_{i}\right\rangle+\sum_{i}\left\langle\nabla_{e_{i}}\left(A_{2}\left(H_{1}, H_{2}\right)\right), e_{i}\right\rangle \\
& +\sum_{\alpha}\left\langle\nabla_{e_{\alpha}}\left(A_{1}\left(H_{2}, H_{1}\right)\right), e_{\alpha}\right\rangle+\sum_{\alpha}\left\langle\nabla_{e_{\alpha}}\left(A_{2}\left(H_{1}, H_{2}\right)\right), e_{\alpha}\right\rangle \\
= & \sum_{i}\left\langle\nabla_{e_{i}}\left(A_{1}\left(H_{2}, H_{1}\right)\right), e_{i}\right\rangle+\sum_{\alpha}\left\langle\nabla_{e_{\alpha}}\left(A_{2}\left(H_{1}, H_{2}\right)\right), e_{\alpha}\right\rangle \\
& +\sum_{i}\left[e_{i}\left\langle A_{2}\left(H_{1}, H_{2}\right), e_{i}\right\rangle-\left\langle A_{2}\left(H_{1}, H_{2}\right), \nabla_{e_{i}} e_{i}\right\rangle\right] \\
& +\sum_{\alpha}\left[e_{\alpha}\left\langle A_{1}\left(H_{2}, H_{1}\right), e_{\alpha}\right\rangle-\left\langle A_{1}\left(H_{2}, H_{1}\right), \nabla_{e_{\alpha}} e_{\alpha}\right\rangle\right], \\
\operatorname{div} Z_{1}= & \sum_{i}\left\langle\nabla_{e_{i}}\left(A_{1}\left(H_{2}, H_{1}\right)\right), e_{i}\right\rangle+\sum_{\alpha}\left\langle\nabla_{e_{\alpha}}\left(A_{2}\left(H_{1}, H_{2}\right)\right), e_{\alpha}\right\rangle \\
& -\sum_{i}\left[\left\langle A_{2}\left(H_{1}, H_{2}\right),\left(\nabla_{e_{i}} e_{i}\right)^{\perp}\right\rangle+\left\langle A_{2}\left(H_{1}, H_{2}\right),\left(\nabla_{e_{i}} e_{i}\right)^{\top}\right\rangle\right] \\
& -\sum_{\alpha}\left[\left\langle A_{1}\left(H_{2}, H_{1}\right),\left(\nabla_{e_{\alpha}} e_{\alpha}\right)^{\top}\right\rangle+\left\langle A_{1}\left(H_{2}, H_{1}\right),\left(\nabla_{e_{\alpha}} e_{\alpha}\right)^{\perp}\right\rangle\right]
\end{aligned}
$$




$$
\begin{aligned}
= & \sum_{i}\left\langle\nabla_{e_{i}}\left(A_{1}\left(H_{2}, H_{1}\right)\right), e_{i}\right\rangle+\sum_{\alpha}\left\langle\nabla_{e_{\alpha}}\left(A_{2}\left(H_{1}, H_{2}\right)\right), e_{\alpha}\right\rangle \\
& -\left\langle A_{2}\left(H_{1}, H_{2}\right), \sum_{i}\left(\nabla_{e_{i}} e_{i}\right)^{\perp}\right\rangle-\left\langle A_{1}\left(H_{2}, H_{1}\right), \sum_{\alpha}\left(\nabla_{e_{\alpha}} e_{\alpha}\right)^{\top}\right\rangle
\end{aligned}
$$

and

$$
\begin{aligned}
\operatorname{div} Z_{1}= & \sum_{i}\left\langle\left(\nabla_{e_{i}} A_{1}\right)\left(H_{2}, H_{1}\right), e_{i}\right\rangle+\sum_{\alpha}\left\langle\left(\nabla_{e_{\alpha}} A_{2}\right)\left(H_{1}, H_{2}\right), e_{\alpha}\right\rangle \\
& -\left\langle A_{2}\left(H_{1}, H_{2}\right), H_{1}\right\rangle-\left\langle A_{1}\left(H_{2}, H_{1}\right), H_{2}\right\rangle .
\end{aligned}
$$

Next, for the second fundamental form $B$ of an arbitrary distribution $D$ (in particular, $D=D_{1}$ or $D=D_{2}$ ) and arbitrary vectors $X, Y, Z \in D$, applying the connections (all of them being denoted by $\nabla$ ) induced by the Levi-Civita connection on $M$ in different bundles $\left(D, D^{\perp}\right.$ etc.) we get

$$
\begin{aligned}
& \left(\nabla_{X} B\right)(Y, Z)-\left(\nabla_{Y} B\right)(X, Z) \\
& =\frac{1}{2}\left(\left(\nabla_{X} \nabla_{Y} Z\right)^{\perp}-\left(\nabla_{Y} \nabla_{X} Z\right)^{\perp}-\left(\nabla_{[X, Y]^{\top}} Z\right)^{\perp}-\left(\nabla_{Z}[X, Y]^{\top}\right)^{\perp}\right. \\
& +\left(\nabla_{X}\left(\nabla_{Z} Y\right)^{\perp}\right)^{\perp}-\left(\nabla_{Y}\left(\nabla_{Z} X\right)^{\perp}\right)^{\perp}
\end{aligned}
$$

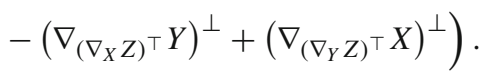

Applying the identity $[X, Y]=\nabla_{X} Y-\nabla_{Y} X$, we obtain the relations

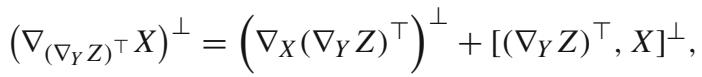

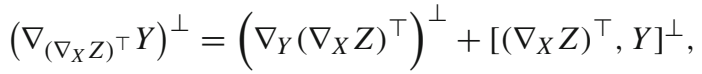

$$
\begin{aligned}
& \left(\nabla_{X}\left(\nabla_{Z} Y\right)^{\perp}\right)^{\perp}=\left[\nabla_{X}\left(\left(\nabla_{Y} Z\right)^{\perp}+[Z, Y]^{\perp}\right)\right]^{\perp}, \\
& \left(\nabla_{Y}\left(\nabla_{Z} X\right)^{\perp}\right)^{\perp}=\left[\nabla_{Y}\left(\left(\nabla_{X} Z\right)^{\perp}+[Z, X]^{\perp}\right)\right]^{\perp}, \\
& \frac{1}{2}\left[\nabla_{Z}\left([X, Y]^{\top}\right)\right]^{\perp}=\frac{1}{2}\left(\nabla_{[X, Y]^{\top}} Z\right)^{\perp}+T\left(Z,\left(\nabla_{X} Y\right)^{\top}\right)-T\left(Z,\left(\nabla_{Y} X\right)^{\top}\right) \text {. }
\end{aligned}
$$

Comparing equalities (6)-(7) with the definition of the curvature tensor $R$ we obtain the following

Proposition 1 For any vectors $X, Y$ and $Z$ belonging to a distribution $D$ on a Riemannian manifold $M$ on has

$$
\begin{aligned}
& \left(\nabla_{X} B\right)(Y, Z)-\left(\nabla_{Y} B\right)(X, Z) \\
& \quad=(R(X, Y), Z)^{\perp}+2\left(\nabla_{T(X, Y)} Z\right)^{\perp}+\left(\nabla_{X} T\right)(Z, Y)-\left(\nabla_{Y} T\right)(Z, X) .
\end{aligned}
$$

Equation (12) can be considered as the Codazzi equation for $D$, an analog of the Codazzi equation for surfaces and arbitrary submanifolds of Riemannian spaces. 
Coming back to our pair of distributions $D_{1}$ and $D_{2}$, we observe that for the operator $A_{1}: D_{1} \times D_{2} \rightarrow D_{1}$, vector fields $X, Y, Z$ in $D_{1}$ and $N$ in $D_{2}$ we have

$$
\begin{aligned}
& \left\langle\nabla_{X} A_{1}(Y, N), Z\right\rangle=X\left\langle A_{1}(Y, N), Z\right\rangle-\left\langle A_{1}(Y, N),\left(\nabla_{X} Z\right)^{\top}\right\rangle \\
& =\left\langle\left(\nabla_{X} B_{1}(Y, Z)\right)^{\perp}, N\right\rangle+\left\langle B_{1}(Y, Z),\left(\nabla_{X} N\right)^{\perp}\right\rangle-\left\langle B_{1}\left(Y,\left(\nabla_{X} Z\right)^{\top}\right), N\right\rangle
\end{aligned}
$$

and similarly

$$
\begin{aligned}
& \left\langle\nabla_{Y} A_{1}(X, N), Z\right\rangle \\
& \quad=\left\langle\left(\nabla_{Y} B_{1}(X, Z)\right)^{\perp}, N\right\rangle+\left\langle B_{1}(X, Z),\left(\nabla_{Y} N\right)^{\perp}\right\rangle-\left\langle B_{1}\left(X,\left(\nabla_{Y} N\right)^{\top}\right), N\right\rangle .
\end{aligned}
$$

Applying the Codazzi equation to the operator $B_{1}$ and comparing equalities (12), (13), (14), we obtain another equation for the operator $A_{1}$ and vector fields $X, Y, Z$ in $D_{1}$ and $N$ in $D_{2}$ :

$$
\begin{aligned}
\left\langle\left(\nabla_{X} A_{1}\right)(Y, N), Z\right\rangle-\left\langle\left(\nabla_{Y} A_{1}\right)(X, N), Z\right\rangle \\
=\left\langle(R(X, Y) Z)^{\perp}+2\left(\nabla_{T_{1}(X, Y)} Z\right)^{\perp}+\left(\nabla_{X} T_{1}\right)(Z, Y)-\left(\nabla_{Y} T_{1}\right)(Z, X), N\right\rangle \\
=\left\langle(R(X, Y) Z)^{\perp}, N\right\rangle+2\left\langle\left(\nabla_{T_{1}(X, Y)} Z\right)^{\perp}, N\right\rangle \\
\quad+\left\langle\left(\nabla_{X} T_{1}\right)(Z, Y)^{\perp}, N\right\rangle-\left\langle\left(\nabla_{Y} T_{1}\right)(Z, X)^{\perp}, N\right\rangle .
\end{aligned}
$$

For the second fundamental form $B_{2}: D_{2} \times D_{2} \rightarrow D_{1}$, operator $A_{2}: D_{2} \times D_{1} \rightarrow D_{2}$, $X^{\prime}, Y^{\prime}, Z^{\prime}$ in $D_{2}$ and $N^{\prime}$ in $D_{1}$ we get similarly

$$
\begin{aligned}
& \left(\nabla_{X^{\prime}} B_{2}\right)\left(Y^{\prime}, Z^{\prime}\right)-\left(\nabla_{Y^{\prime}} B_{2}\right)\left(X^{\prime}, Z^{\prime}\right) \\
& \quad=\left(R\left(X^{\prime}, Y^{\prime}\right) Z^{\prime}\right)^{\top}+2\left(\nabla_{T_{2}\left(X^{\prime}, Y^{\prime}\right)} Z^{\prime}\right)^{\top}+\left(\nabla_{X^{\prime}} T_{2}\right)\left(Z^{\prime}, Y^{\prime}\right)-\left(\nabla_{Y} T_{2}\right)\left(Z^{\prime}, X^{\prime}\right)
\end{aligned}
$$

and

$$
\begin{aligned}
& \left\langle\left(\nabla_{X^{\prime}} A_{2}\right)\left(Y^{\prime}, N^{\prime}\right), Z^{\prime}\right\rangle-\left\langle\left(\nabla_{Y^{\prime}} A_{2}\right)\left(X^{\prime}, N^{\prime}\right), Z^{\prime}\right\rangle=\left\langle\left(R\left(X^{\prime}, Y^{\prime}\right) Z^{\prime}\right)^{\top}, N^{\prime}\right\rangle \\
& +2\left\langle\left(\nabla_{T_{2}\left(X^{\prime}, Y^{\prime}\right)} Z^{\prime}\right)^{\top}, N^{\prime}\right\rangle+\left\langle\left(\nabla_{X^{\prime}} T_{2}\right)\left(Z^{\prime}, Y^{\prime}\right)^{\top}, N^{\prime}\right\rangle-\left\langle\left(\nabla_{Y^{\prime}} T_{2}\right)\left(Z^{\prime}, X^{\prime}\right)^{\top}, N^{\prime}\right\rangle .
\end{aligned}
$$

Moreover, applying Eq. (15) to $X=Z=e_{i}, Y=H_{2}$ and $N=H_{1}$ we obtain consecutively

$$
\begin{aligned}
\sum_{i} & \left\langle\nabla_{e_{i}} A_{1}\left(H_{2}, H_{1}\right), e_{i}\right\rangle \\
= & \sum_{i}\left(\left\langle\left(\nabla_{e_{i}} A_{1}\right)\left(H_{2}, H_{1}\right), e_{i}\right\rangle+\left\langle A_{1}\left(\left(\nabla_{e_{i}} H_{2}\right)^{\top}, H_{1}\right), e_{i}\right\rangle+\left\langle A_{1}\left(H_{2},\left(\nabla_{e_{i}} H_{1}\right)^{\perp}\right), e_{i}\right\rangle\right) \\
= & \sum_{i}\left(\left\langle\left(\nabla_{H_{2}} A_{1}\right)\left(e_{i}, H_{1}\right), e_{i}\right\rangle+\left\langle\left(R\left(e_{i}, H_{2}\right) e_{i}\right)^{\perp}, H_{1}\right\rangle+2\left\langle\left(\nabla_{T_{1}\left(e_{i}, H_{2}\right)} e_{i}\right)^{\perp}, H_{1}\right\rangle\right. \\
& +\left\langle\left(\nabla_{e_{i}} T_{1}\right)\left(e_{i}, H_{2}\right)^{\perp}, H_{1}\right\rangle-\left\langle\left(\nabla_{H_{2}} T_{1}\right)\left(e_{i}, e_{i}\right)^{\perp}, H_{1}\right\rangle \\
& \left.+\left\langle A_{1}\left(\left(\nabla_{e_{i}} H_{2}\right)^{\top}, H_{1}\right), e_{i}\right\rangle+\left\langle A_{1}\left(H_{2},\left(\nabla_{e_{i}} H_{1}\right)^{\perp}\right), e_{i}\right\rangle\right)
\end{aligned}
$$




$$
\begin{aligned}
= & \sum_{i}\left(\left\langle\nabla_{H_{2}} A_{1}\left(e_{i}, H_{1}\right), e_{i}\right\rangle-\left\langle A_{1}\left(\left(\nabla_{H_{2}} e_{i}\right)^{\top}, H_{1}\right), e_{i}\right\rangle-\left\langle A_{1}\left(e_{i},\left(\nabla_{H_{2}} H_{1}\right)^{\perp}\right), e_{i}\right\rangle\right. \\
& +\left\langle\left(R\left(e_{i}, H_{2}\right) e_{i}\right)^{\perp}, H_{1}\right\rangle+2\left\langle\left(\nabla_{T_{1}\left(e_{i}, H_{2}\right)} e_{i}\right)^{\perp}, H_{1}\right\rangle \\
& \left.+\left\langle\left(\nabla_{e_{i}} T_{1}\right)\left(e_{i}, H_{2}\right)^{\perp}, H_{1}\right\rangle+\left\langle A_{1}\left(\left(\nabla_{e_{i}} H_{2}\right)^{\top}, H_{1}\right), e_{i}\right\rangle+\left\langle A_{1}\left(H_{2},\left(\nabla_{e_{i}} H_{1}\right)^{\perp}\right), e_{i}\right\rangle\right), \\
& \sum_{i}\left\langle\nabla_{e_{i}} A_{1}\left(H_{2}, H_{1}\right), e_{i}\right\rangle \\
= & \sum_{i}\left(H_{2}\left\langle A_{1}\left(e_{i}, H_{1}\right), e_{i}\right\rangle-\left\langle A_{1}\left(e_{i}, H_{1}\right),\left(\nabla_{H_{2}} e_{i}\right)^{\top}\right\rangle-\left\langle B_{1}\left(\left(\nabla_{H_{2}} e_{i}\right)^{\top}, e_{i}\right), H_{1}\right\rangle\right. \\
& -\left\langle B_{1}\left(e_{i}, e_{i}\right),\left(\nabla_{H_{2}} H_{1}\right)^{\perp}\right\rangle+\left\langle A_{1}\left(\left(\nabla_{e_{i}} H_{2}\right)^{\top}, H_{1}\right), e_{i}\right\rangle+\left\langle A_{1}\left(H_{2},\left(\nabla_{e_{i}} H_{1}\right)^{\perp}\right), e_{i}\right\rangle \\
& \left.+\left\langle\left(R\left(e_{i}, H_{2}\right) e_{i}\right)^{\perp}, H_{1}\right\rangle+2\left\langle\left(\nabla_{T_{1}\left(e_{i}, H_{2}\right)} e_{i}\right)^{\perp}, H_{1}\right\rangle+\left\langle\left(\nabla_{e_{i}} T_{1}\right)\left(e_{i}, H_{2}\right)^{\perp}, H_{1}\right\rangle\right)
\end{aligned}
$$

and

$$
\begin{aligned}
\sum_{i} & \left\langle\nabla_{e_{i}} A_{1}\left(H_{2}, H_{1}\right), e_{i}\right\rangle \\
= & H_{2}\left\|H_{1}\right\|^{2}-\left\langle H_{1},\left(\nabla_{H_{2}} H_{1}\right)^{\perp}\right\rangle \\
& +\sum_{i}\left(\left\langle\left(R\left(e_{i}, H_{2}\right) e_{i}\right)^{\perp}, H_{1}\right\rangle+2\left\langle\left(\nabla_{T_{1}\left(e_{i}, H_{2}\right)} e_{i}\right)^{\perp}, H_{1}\right\rangle\right. \\
& +\left\langle\left(\nabla_{e_{i}} T_{1}\right)\left(e_{i}, H_{2}\right)^{\perp}, H_{1}\right\rangle-2\left\langle B_{1}\left(\left(\nabla_{H_{2}} e_{i}\right)^{\top}, e_{i}\right), H_{1}\right\rangle \\
& \left.+\left\langle A_{1}\left(\left(\nabla_{e_{i}} H_{2}\right)^{\top}, H_{1}\right), e_{i}\right\rangle+\left\langle A_{1}\left(H_{2},\left(\nabla_{e_{i}} H_{1}\right)^{\perp}\right), e_{i}\right\rangle\right) .
\end{aligned}
$$

Analogously, applying Eq. (17) to $X^{\prime}=Z^{\prime}=e_{\alpha}, Y^{\prime}=H_{1}$ and $N^{\prime}=H_{2}$ we get

$$
\begin{aligned}
& \sum_{\alpha}\left\langle\nabla_{e_{\alpha}} A_{2}\left(H_{1}, H_{2}\right), e_{\alpha}\right\rangle=H_{1}\left\|H_{2}\right\|^{2}-\left\langle H_{2},\left(\nabla_{H_{1}} H_{2}\right)^{\top}\right\rangle \\
& \quad+\sum_{\alpha}\left(\left\langle\left(R\left(e_{\alpha}, H_{1}\right) e_{\alpha}\right)^{\top}, H_{2}\right\rangle+2\left\langle\left(\nabla_{T_{2}\left(e_{\alpha}, H_{1}\right)} e_{\alpha}\right)^{\top}, H_{2}\right\rangle\right. \\
& \quad+\left\langle\left(\nabla_{e_{\alpha}} T_{2}\right)\left(e_{\alpha}, H_{1}\right)^{\top}, H_{2}\right\rangle-2\left\langle B_{2}\left(\left(\nabla_{H_{1}} e_{\alpha}\right)^{\perp}, e_{\alpha}\right), H_{2}\right\rangle \\
& \left.\quad+\left\langle A_{2}\left(\left(\nabla_{e_{\alpha}} H_{1}\right)^{\perp}, H_{2}\right), e_{\alpha}\right\rangle+\left\langle A_{2}\left(H_{1},\left(\nabla_{e_{\alpha}} H_{2}\right)^{\top}\right), e_{\alpha}\right\rangle\right) .
\end{aligned}
$$

Next, let us observe that

$$
\begin{aligned}
\sum_{i}\left\langle A_{1}\left(\left(\nabla_{e_{i}} H_{2}\right)^{\top}, H_{1}\right), e_{i}\right\rangle & =\sum_{i, j}\left\langle A_{i}\left(e_{j}, H_{1}\right), e_{i}\right\rangle\left\langle e_{,}\left(\nabla_{e_{i}} H_{2}\right)^{\top}\right\rangle \\
& =\sum_{i, j}\left\langle A_{i}\left(e_{i}, H_{1}\right), e_{j}\right\rangle\left\langle e_{j},\left(\nabla_{e_{i}} H_{2}\right)^{\top}\right\rangle \\
& =\left\langle A_{1}^{H_{1}}, \nabla_{\bullet}^{\top} H_{2}\right\rangle
\end{aligned}
$$


and similarly

$$
\begin{aligned}
\sum_{\alpha}\left\langle A_{2}\left(\left(\nabla_{e_{\alpha}} H_{1}\right)^{\perp}, H_{2}\right), e_{\alpha}\right\rangle & =\sum_{\alpha, \beta}\left\langle A_{2}\left(e_{\alpha}, H_{2}\right), e_{\beta}\right\rangle\left\langle e_{\beta},\left(\nabla_{e_{\alpha}} H_{1}\right)^{\perp}\right\rangle \\
& =\left\langle A_{2}^{H_{2}}, \nabla_{\bullet}^{\perp} H_{2}\right\rangle .
\end{aligned}
$$

The well-known properties of the curvature tensor $R$ imply the equality

$$
\sum_{i}\left\langle\left(R\left(e_{i}, H_{2}\right) e_{i}\right)^{\perp}, H_{1}\right\rangle+\sum_{\alpha}\left\langle\left(R\left(e_{\alpha}, H_{1}\right) e_{\alpha}\right)^{\top}, H_{2}\right\rangle=\left\langle\operatorname{Ric}\left(H_{2}\right), H_{1}\right\rangle .
$$

Finally, it is easy to show that

$$
\sum_{i}\left\langle A_{1}^{H_{1}}\left(\nabla_{H_{2}} e_{i}\right)^{\top}, e_{i}\right\rangle=0 \text { and } \sum_{\alpha}\left\langle A_{2}^{H_{2}}\left(\nabla_{H_{1}} e_{\alpha}\right)^{\perp}, e_{\alpha}\right\rangle=0 .
$$

Applying Eqs. (18)-(23) to (5) we end up with the following

Proposition 2 In the situation considered above, one has

$$
\begin{aligned}
\operatorname{div} & \left(A_{1}\left(H_{2}, H_{1}\right)+A_{2}\left(H_{1}, H_{2}\right)\right) \\
= & \left\langle\operatorname{Ric}\left(H_{2}\right), H_{1}\right\rangle+\left\langle H_{1},\left(\nabla_{H_{2}} H_{1}\right)^{\perp}\right\rangle+\left\langle H_{2},\left(\nabla_{H_{1}} H_{2}\right)^{\top}\right\rangle \\
& +\left\langle\operatorname{Tr}^{\perp}\left(\nabla \cdot T_{1}\right)\left(\cdot, H_{2}\right), H_{1}\right\rangle+\left\langle\operatorname{Tr}^{\top}\left(\nabla . T_{2}\right)\left(\cdot, H_{1}\right), H_{2}\right\rangle \\
& +\left\langle A_{1}^{H_{1}}, \nabla^{\top} H_{2}\right\rangle+\left\langle A_{2}^{H_{2}}, \nabla^{\perp} H_{2}\right\rangle \\
& +\sum_{i}\left\langle A_{1}\left(H_{2},\left(\nabla_{e_{i}} H_{1}\right)^{\perp}\right), e_{i}\right\rangle+\sum_{\alpha}\left\langle A_{2}\left(H_{1},\left(\nabla_{e_{\alpha}} H_{2}\right)^{\top}\right), e_{\alpha}\right\rangle \\
& +2 \sum_{i}\left\langle\left(\nabla_{T_{1}\left(e_{i}, H_{2}\right)} e_{i}\right)^{\perp}, H_{1}\right\rangle+2 \sum_{\alpha}\left\langle\left(\nabla_{T_{2}\left(e_{\alpha}, H_{1}\right)} e_{\alpha}\right)^{\top}, H_{2}\right\rangle \\
& -\left\langle A_{2}\left(H_{1}, H_{2}\right), H_{1}\right\rangle-\left\langle A_{1}\left(H_{2}, H_{1}\right), H_{2}\right\rangle .
\end{aligned}
$$

Integrating (24) and applying the Stokes Theorem, we get our integral formula:

Theorem 1 For arbitrary orthogonal complementary distributions $D_{1}$ and $D_{2}$ on a closed oriented Riemannian manifold $M$ one has

$$
\begin{aligned}
& \int_{M}\left(\left\langle\operatorname{Ric}\left(H_{2}\right), H_{1}\right\rangle+\left\langle H_{1},\left(\nabla_{H_{2}} H_{1}\right)^{\perp}\right\rangle+\left\langle H_{2},\left(\nabla_{H_{1}} H_{2}\right)^{\top}\right\rangle\right. \\
& \quad+\left\langle\operatorname{Tr}^{\perp}\left(\nabla . T_{1}\right)\left(\cdot, H_{2}\right), H_{1}\right\rangle+\left\langle\operatorname{Tr}^{\top}\left(\nabla . T_{2}\right)\left(\cdot, H_{1}\right), H_{2}\right\rangle+\left\langle A_{1}^{H_{1}}, \nabla^{\top} H_{2}\right\rangle \\
& \quad+\left\langle A_{2}^{H_{2}}, \nabla_{\cdot}^{\perp} H_{2}\right\rangle+\sum_{i}\left\langle A_{1}\left(H_{2},\left(\nabla_{e_{i}} H_{1}\right)^{\perp}\right), e_{i}\right\rangle+\sum_{\alpha}\left\langle A_{2}\left(H_{1},\left(\nabla_{e_{\alpha}} H_{2}\right)^{\top}\right), e_{\alpha}\right\rangle \\
& \quad+2 \sum_{i}\left\langle\left(\nabla_{T_{1}\left(e_{i}, H_{2}\right)} e_{i}\right)^{\perp}, H_{1}\right\rangle+2 \sum_{\alpha}\left\langle\left(\nabla_{T_{2}\left(e_{\alpha}, H_{1}\right)} e_{\alpha}\right)^{\top}, H_{2}\right\rangle \\
& \left.\quad-\left\langle A_{2}\left(H_{1}, H_{2}\right), H_{1}\right\rangle-\left\langle A_{1}\left(H_{2}, H_{1}\right), H_{2}\right\rangle\right) d \mathrm{vol}=0 .
\end{aligned}
$$




\section{Distributions with singularities}

In this section, we work with a closed Riemannian manifold $M$ equipped with a pair of orthogonal and complementary distributions $\left(D_{1}, D_{2}\right)$ defined on $M \backslash \Sigma, \Sigma$ being the union of pairwise disjoint closed submanifolds of variable codimensions $\geq 2$. Briefly, we say that our distributions admit singularities at points of $\Sigma$. We shall show that in this case, the integral formulae (4) and (25) hold under some natural assumptions.

First, observe that $M$ has bounded geometry, i.e., bounded sectional curvature and injectivity radii $r_{x}, x \in M$, separated away from zero. Let $A$ be a closed submanifold of $M$ and $k=\operatorname{codim} A \geq 2$. Given $r>0$, we denote the tube of radius $r$ about $A$ by $N_{A}(r)$ and by $\partial N_{A}(r)$ the tubular hypersurface at distance $r \geq 0$ from $A$. Let $f: M \backslash A \rightarrow[0,+\infty)$ be a function defined on $M$ outside $A$.

Lemma 1 If $\liminf _{r \rightarrow 0^{+}} \int_{\partial N_{A}(r)} f>0$, then $\int_{M} f^{2}=\infty$.

Proof Since the geometry of $M$ is bounded, there exists $c>0$ such that $\operatorname{vol}\left(\partial N_{A}(r)\right) \leq$ $c \cdot r^{k-1}$ for sufficiently small $r$. The assumption implies that there exists $\varepsilon>0$ such that

$$
\int_{\partial N_{A}} f \geq \varepsilon
$$

for small $r$. Hölder's inequality implies that

$$
\int_{\partial N_{A}(r)} f \leq\left(\int_{\partial N_{A}(r)} f^{2}\right)^{\frac{1}{2}} \cdot \operatorname{vol}\left(\partial N_{A}(r)\right)^{\frac{1}{2}} .
$$

Consequently,

$$
\int_{\partial N_{A}(r)} f^{2} \geq \frac{\varepsilon^{2}}{c \cdot r^{k-1}}
$$

if $r$ is small enough.

Again, if $r$ is small, then by Fubini's Theorem,

$$
\int_{M} f^{2} \geq \int_{N_{A}(r)} f^{2}=\int_{0}^{r}\left(\int_{\partial N_{A}(t)} f^{2}\right) \mathrm{d} t \geq \frac{\varepsilon^{2}}{c} \lim _{\xi \rightarrow 0^{+}} \int_{\xi}^{r} t^{1-k}=\infty .
$$

The above implies the following.

Lemma 2 If $Z$ is a vector field on $M \backslash A$ such that $\int_{M}\|Z\|^{2}<\infty$, then

$$
\int_{M} \operatorname{div} Z=0
$$

Proof Let $v_{r}$ be the suitably oriented unit vector field orthogonal to $\partial N_{A}(r)$. By the Stokes Theorem and our Lemma 1 applied to $f=\|Z\|$, we get

$$
\int_{M \backslash N_{A}(r)} \operatorname{div} Z=\int_{\partial N_{A}(r)}\left\langle Z, v_{r}\right\rangle \leq \int_{\partial N_{A}(r)}\|Z\| \rightarrow 0
$$

as $r \rightarrow 0$.

Applying Lemma 2 to $Z=Z_{k}(k=0,1)$ we get 
Theorem 2 Let $M$ be closed and oriented and distributions $D_{1}$ and $D_{2}$ be defined on $M \backslash \Sigma$, $\operatorname{codim} \Sigma \geq 2$.

(i) If $\int_{M}\left\|H_{1}\right\|^{2}<\infty$ and $\int_{M}\left\|H_{2}\right\|^{2}<\infty$, then formula (4) holds.

(ii) If $\int_{M}\left\|A_{1}\left(H_{2}, H_{1}\right)\right\|^{2}<\infty$ and $\int_{M}\left\|A_{2}\left(H_{1}, H_{2}\right)\right\|^{2}<\infty$, then formula (25) holds.

Finally, let us observe that since $\left\|H_{i}\right\| \leq c(p, q) \cdot\left\|A_{i}\right\|$ for a constant $c(p, q)$ depending on $p$ and $q$ only, the inequalities in (ii) above hold for example when $\int_{M}\left\|A_{i}\right\|^{6}<\infty$ for $i=1$, 2. Indeed, for, say, $i=1$, we have

$$
\left\|A_{1}\left(H_{2}, H_{1}\right)\right\| \leq\left\|A_{1}\right\| \cdot\left\|H_{1}\right\| \cdot\left\|H_{2}\right\| \leq c(p, q)^{2}\left\|A_{1}\right\|^{2} \cdot\left\|A_{2}\right\|
$$

and, by the Hölder inequality,

$$
\int_{M}\left\|A_{1}\left(H_{2}, H_{1}\right)\right\|^{2} \leq c(p, q)^{4} \cdot\left(\int_{M}\left\|A_{1}\right\|^{6}\right)^{2 / 3} \cdot\left(\int_{M}\left\|A_{2}\right\|^{6}\right)^{1 / 3} .
$$

\section{Applications}

In this section, we will consider pairs of distributions $D_{i}, i=1,2$, satisfying some geometrical conditions, write formulae (4) and (25) in particular cases and prove some (non-)existence results which follow from them. Our distributions are defined either on a closed manifold $M$ or on $M \backslash \Sigma, \Sigma$ being the union of a finite family of embedded closed submanifolds of codimension $\geq 2$.

\subsection{Minimal and totally geodesic distributions}

First, if $D_{1}$ and $D_{2}$ are totally geodesic, that is $B_{1}=0$ and $B_{2}=0$, then $H_{1}=0, H_{2}=0$ and (3) reduces to the identity

$$
K\left(D_{1}, D_{2}\right)-\left\|T_{1}\right\|^{2}-\left\|T_{2}\right\|^{2}=0
$$

which implies immediately the analogous equality for the integral in (4) and the following.

Corollary 1 If a closed manifold $M$ has non-positive sectional curvature $\left(K_{M} \leq 0\right)$ and admits a pair of orthogonal complementary totally geodesic distributions, then $M$ is flat $\left(K_{M} \equiv 0\right)$.

If $D_{1}$ and $D_{2}$ are minimal, that is $H_{1}=0$ and $H_{2}=0$, and integrable $\left(T_{1}=0\right.$ and $T_{2}=0$ ), then (4) reduces to the identity

$$
K\left(D_{1}, D_{2}\right)+\left\|B_{1}\right\|^{2}+\left\|B_{2}\right\|^{2}=0 .
$$

As before, this implies the analogous integral equality and the following.

Corollary 2 If a closed manifold $M$ has non-negative sectional curvature and admits a pair of orthogonal complementary minimal distributions, then $M$ is flat.

In both cases, this of totally geodesic and that of minimal (either integrable or not) distributions (25) reduces to the identity " $0=0$ ", so yields no reasonable consequences. 


\subsection{Umbilical distributions}

Let us recall that a distribution $D$ is said to be umbilical, when its Weingarten operator $A$ satisfies

$$
A(X, N)=\omega(N) \cdot X,
$$

for any $X \in D$ and $N \perp D$, and some 1-form $\omega$.

Assume now that our pair $\left(D_{1}, D_{2}\right)$ consists of two umbilical distributions, one of them, say $D_{1}$, being integrable and denote by $\omega_{i}, i=1,2$, corresponding 1 -forms. Note that, this situation is of some interest: umbilicity is a conformally invariant property, the distribution orthogonal to a Hopf fibration on the round sphere $S^{2 n+1}$ is totally geodesic [so, umbilical with $\omega=0$ in (26)], fibers are also totally geodesics (so, umbilical), therefore, Hopf fibrations provide pairs of umbilical distributions, one of them being integrable, on odd-dimensional spheres equipped with arbitrary locally conformally flat Riemannian structures.

In the situation considered here, we obtain

$$
\begin{aligned}
\left\langle H_{1}, N\right\rangle & =\sum_{i}\left\langle B_{1}\left(e_{i}, e_{i}\right), N\right\rangle=\sum_{i}\left\langle A_{1}\left(e_{i}, N\right), e_{i}\right\rangle \\
& =\sum_{i} \omega_{1}(N)\left\langle e_{i}, e_{i}\right\rangle=p \omega(N), \\
A_{1}(X, N) & =\frac{1}{p}\left\langle H_{1}, N\right\rangle \cdot X,
\end{aligned}
$$

and

$$
\left\langle B_{1}(X, Y), N\right\rangle=\left\langle A_{1}(X, N), Y\right\rangle=\frac{1}{p}\left\langle H_{1}, N\right\rangle \cdot\langle X, Y\rangle
$$

for $X, Y \in D_{1}$ and $N \in D_{2}$. Similarly, for $D_{2}$ and vectors $X, Y \in D_{2}$ and $N \in D_{1}$ we get

$$
A_{2}(Y, N)=\frac{1}{q}\left\langle H_{2}, N\right\rangle \cdot Y
$$

and

$$
\left\langle B_{2}(X, Y), N\right\rangle=\left\langle A_{2}(X, N), Y\right\rangle=\frac{1}{q}\left\langle H_{2}, N\right\rangle \cdot\langle X, Y\rangle .
$$

Applying the above equalities, we obtain

$$
\begin{aligned}
& \left\langle A_{1}^{H_{1}}, \nabla^{\top} H_{2}\right\rangle \\
& =\sum_{i}\left\langle A_{1}\left(e_{i}, H_{1}\right), \nabla_{e_{i}}^{\top} H_{2}\right\rangle=\frac{1}{p} \sum_{i}\left\langle e_{i}, \nabla_{e_{i}} H_{2}\right\rangle\left\langle H_{1}, H_{1}\right\rangle \\
& =\frac{1}{p}\left\|H_{1}\right\|^{2} \cdot \text { Trace } \nabla^{\top} H_{2}
\end{aligned}
$$


and

$$
\begin{aligned}
& \sum_{i}\left\langle A_{1}\left(H_{2},\left(\nabla_{e_{i}} H_{1}\right)^{\perp}\right), e_{i}\right\rangle \\
& =\frac{1}{p} \sum_{i}\left\langle e_{i}, H_{2}\right\rangle\left\langle\nabla_{e_{i}} H_{1}, H_{1}\right\rangle=\left\langle\nabla_{\sum_{i}<e_{i}, H_{2}>e_{i}} H_{1}, H_{1}\right\rangle \\
& =\frac{1}{p}\left\langle\nabla_{H_{2}} H_{1}, H_{1}\right\rangle .
\end{aligned}
$$

Similarly,

$$
\left\langle A_{2}^{H_{2}}, \nabla^{\perp} H_{1}\right\rangle=\frac{1}{q}\left\|H_{2}\right\|^{2} \cdot \operatorname{Tr} \nabla^{\perp} H_{1}
$$

and

$$
\sum_{\alpha}\left\langle A_{2}\left(H_{1},\left(\nabla_{e_{\alpha}} H_{2}\right)^{\perp}\right), e_{\alpha}\right\rangle=\frac{1}{q}\left\langle\nabla_{H_{1}} H_{2}, H_{2}\right\rangle .
$$

Moreover,

$\left\langle A_{1}\left(H_{2}, H_{1}\right), H_{2}\right\rangle=\frac{1}{p}\left\|H_{1}\right\|^{2} \cdot\left\|H_{2}\right\|^{2}$ and $\left\langle A_{2}\left(H_{1}, H_{2}\right), H_{1}\right\rangle=\frac{1}{q}\left\|H_{2}\right\|^{2} \cdot\left\|H_{1}\right\|^{2}$.

Finally, since $D_{1}$ is integrable, $T_{1}=0$ and formulae (24) and (25) reduce, respectively, to

$$
\begin{aligned}
\operatorname{div} & \left(A_{1}\left(H_{2}, H_{1}\right)+A_{2}\left(H_{1}, H_{2}\right)\right) \\
= & \left\langle\operatorname{Ric}\left(H_{2}\right), H_{1}\right\rangle+2 \sum_{\alpha}\left\langle\left(\nabla_{T_{2}\left(e_{\alpha}, H_{1}\right)} e_{\alpha}\right)^{\top}, H_{2}\right\rangle+\left\langle\operatorname{Tr}^{\top}\left(\nabla \bullet T_{2}\right)\left(\bullet, H_{1}\right), H_{1}\right\rangle \\
& +\frac{1}{p}\left\|H_{1}\right\|^{2} \cdot \operatorname{Tr} \nabla^{\top} H_{2}+\frac{1}{q}\left\|H_{2}\right\|^{2} \cdot \operatorname{Tr} \nabla^{\perp} H_{1} \\
& +\frac{p+1}{p}\left\langle H_{1}, \nabla_{H_{2}} H_{1}\right\rangle+\frac{q+1}{q}\left\langle H_{2}, \nabla_{H_{1}} H_{2}\right\rangle \\
& -\frac{1}{p}\left\|H_{1}\right\|^{2} \cdot\left\|H_{2}\right\|^{2}-\frac{1}{q}\left\|H_{2}\right\|^{2} \cdot\left\|H_{1}\right\|^{2}
\end{aligned}
$$

and (on closed manifolds)

$$
\begin{aligned}
& \int_{M}\left(\left\langle\operatorname{Ric}\left(H_{2}\right), H_{1}\right\rangle+2 \sum_{\alpha}\left\langle\left(\nabla_{T_{2}\left(e_{\alpha}, H_{1}\right)} e_{\alpha}\right)^{\top}, H_{2}\right\rangle+\left\langle\operatorname{Tr}^{\top}\left(\nabla_{\bullet} T_{2}\right)\left(\bullet, H_{1}\right), H_{1}\right\rangle\right. \\
& +\frac{1}{p}\left\|H_{1}\right\|^{2} \cdot \operatorname{Tr} \nabla^{\top} H_{2}+\frac{1}{q}\left\|H_{2}\right\|^{2} \cdot \operatorname{Tr} \nabla^{\perp} H_{1}+\frac{p+1}{p}\left\langle H_{1}, \nabla_{H_{2}} H_{1}\right\rangle \\
& \left.+\frac{q+1}{q}\left\langle H_{2}, \nabla_{H_{1}} H_{2}\right\rangle-\frac{1}{p}\left\|H_{1}\right\|^{2} \cdot\left\|H_{2}\right\|^{2}-\frac{1}{q}\left\|H_{2}\right\|^{2} \cdot\left\|H_{1}\right\|^{2}\right) \mathrm{d} \text { vol }=0 .
\end{aligned}
$$

Also, since

$$
\left\|B_{1}\right\|^{2}=\frac{1}{p} \cdot\left\|H_{1}\right\|^{2} \text { and }\left\|B_{2}\right\|^{2}=\frac{1}{q} \cdot\left\|H_{2}\right\|^{2},
$$

formulae (3) and (4) reduce, respectively, to

$$
\operatorname{div}\left(H_{1}+H_{2}\right)=K\left(D_{1}, D_{2}\right)+\frac{1-p}{p}\left\|H_{1}\right\|^{2}+\frac{1-q}{q}\left\|H_{2}\right\|^{2}-\left\|T_{2}\right\|^{2}
$$


and (on closed manifolds)

$$
\int_{M}\left(K\left(D_{1}, D_{2}\right)+\frac{1-p}{p}\left\|H_{1}\right\|^{2}+\frac{1-q}{q}\left\|H_{2}\right\|^{2}-\left\|T_{2}\right\|^{2}\right) \mathrm{d} \text { vol }=0 .
$$

The last formula above implies the following

Corollary 3 If $K\left(D_{1}, D_{2}\right) \leq 0$ and $p, q>1$, then $H_{1}=H_{2}=0, T_{2}=0$ and $K\left(D_{1}, D_{2}\right)=$ 0 . If $M$ is closed and $K_{M}<0$, then the distributions $D_{1}, D_{2}$ satisfying the conditions of this section do not exist.

The last statement could be compared with Theorem 4.1.2 in [9].

\subsection{Constant mean curvature}

Now, let us recall that a distribution $D$ on a Riemannian manifold $M$ has constant mean curvature whenever its mean curvature vector $H$ satisfies

$$
\nabla^{\perp} H=0,
$$

where $\nabla^{\perp}$ is the connection in $D^{\perp}$ induced by the Levi-Civita connection on $M$.

Coming back to a pair of distributions, let us observe that if both of them, $D_{1}$ and $D_{2}$ have constant mean curvature, then several terms in (24) vanish identically, therefore (24) and (25) reduce, respectively, to

$$
\begin{aligned}
\operatorname{div} & \left(A_{1}\left(H_{2}, H_{1}\right)+A_{2}\left(H_{1}, H_{2}\right)\right) \\
= & \left\langle\operatorname{Ric}\left(H_{2}\right), H_{1}\right\rangle-\left\langle A_{2}\left(H_{1}, H_{2}\right), H_{1}\right\rangle-\left\langle A_{1}\left(H_{2}, H_{1}\right), H_{2}\right\rangle \\
& +\left\langle\operatorname{Trace}^{\perp}\left(\nabla_{\bullet} T_{1}\right)\left(\bullet, H_{2}\right), H_{1}\right\rangle+\left\langle\operatorname{Trace}^{\top}\left(\nabla_{\bullet} T_{2}\right)\left(\bullet, H_{1}\right), H_{2}\right\rangle \\
& +2 \sum_{i}\left\langle\left(\nabla_{T_{1}\left(e_{i}, H_{2}\right)} e_{i}\right)^{\perp}, H_{1}\right\rangle+2 \sum_{\alpha}\left\langle\left(\nabla_{T_{2}\left(e_{\alpha}, H_{1}\right)} e_{\alpha}\right)^{\top}, H_{2}\right\rangle
\end{aligned}
$$

and (on closed manifolds, again)

$$
\begin{aligned}
& \int_{M}\left(\left\langle\operatorname{Ric}\left(H_{2}\right), H_{1}\right\rangle-\left\langle A_{2}\left(H_{1}, H_{2}\right), H_{1}\right\rangle-\left\langle A_{1}\left(H_{2}, H_{1}\right), H_{2}\right\rangle\right. \\
& \quad+\left\langle\operatorname{Trace}^{\perp}\left(\nabla_{\bullet} T_{1}\right)\left(\bullet, H_{2}\right), H_{1}\right\rangle+\left\langle\operatorname{Tr}^{\top}\left(\nabla \bullet T_{2}\right)\left(\bullet, H_{1}\right), H_{2}\right\rangle \\
& \left.\quad+2 \sum_{i}\left\langle\left(\nabla_{T_{1}\left(e_{i}, H_{2}\right)} e_{i}\right)^{\perp}, H_{1}\right\rangle+2 \sum_{\alpha}\left\langle\left(\nabla_{T_{2}\left(e_{\alpha}, H_{1}\right)} e_{\alpha}\right)^{\top}, H_{2}\right\rangle\right) \mathrm{d} \text { vol }=0 .
\end{aligned}
$$

Certainly, there is a number of formulae which can be obtained form (24) and (25) in other geometrically interesting cases. Here, let us mention just the following one.

Proposition 3 If $D_{1}$ and $D_{2}$ are complementary orthogonal distributions on a Riemannian manifold $M$ which are umbilical, integrable and have constant mean curvature, then

$$
\begin{aligned}
\operatorname{div} & \left(A_{1}\left(H_{2}, H_{1}\right)+A_{2}\left(H_{1}, H_{2}\right)\right) \\
= & \left\langle\operatorname{Ric}\left(H_{2}\right), H_{1}\right\rangle-\frac{1}{p}\left\|H_{1}\right\|^{2} \cdot\left\|H_{2}\right\|^{2}-\frac{1}{q}\left\|H_{2}\right\|^{2} \cdot\left\|H_{1}\right\|^{2}
\end{aligned}
$$


and

$$
\int_{M}\left(\left\langle\operatorname{Ric}\left(H_{2}\right), H_{1}\right\rangle-\left(\frac{1}{p}+\frac{1}{q}\right)\left\|H_{1}\right\|^{2} \cdot\left\|H_{2}\right\|^{2}\right) d \operatorname{vol}=0
$$

when $M$ is closed.

Finally, observe that $\left\langle\operatorname{Ric}\left(H_{1}\right), H_{2}\right\rangle=0$ when $M$ is an Einstein manifold. This implies the following application of our main formula (25).

Corollary 4 If $M$ is a closed Einstein manifold, then arbitrary pairs of distributions satisfying the conditions of Proposition 3 have to be totally geodesic.

Proof From (28), we get $H_{1}=H_{2}=0$ what-together with umbilicity-yields $A_{1}=0$ and $A_{2}=0$.

Note that, in the situation described in Corollary $4, M$ is locally isometric to the Riemannian product of the leaves of foliations $\mathcal{F}_{1}$ and $\mathcal{F}_{2}$ determined by the distributions $D_{1}$ and $D_{2}$ of our pair.

\section{An example}

An open book decomposition (OBD, for short) of a three-dimensional manifold $M$ is a pair $(B, \pi)$ where $B$ is an oriented link in $M$, called the binding of the open book, and $\pi: M \backslash B \rightarrow S^{1}$ is a fibration such that, for each $\theta \in S^{1}, \pi^{-1}(\theta)$ is the interior of a compact surface (with boundary) $\Sigma \subset M$ whose boundary is $B$. The surface $\Sigma$ is called the page of the open book. Since almost a century [1], it is known that every closed oriented 3-manifold has an open book decomposition.

A closed 3-manifold $M$ with an open book decomposition $(B, \pi)$ is equipped with two singular distributions $D_{1}$ and $D_{2}\left(\operatorname{dim} D_{1}=2\right.$ and $\left.\operatorname{dim} D_{2}=1\right)$ defined on $M \backslash B$. Both of them are integrable, so $T_{1}=0$ and $T_{2}=0$, and $\left\|B_{2}\right\|^{2}=\left\|H_{2}\right\|^{2}$. Therefore, (4) reduces to (1) (for the foliation of $M \backslash B$ by the pages of the open book) which holds here if only the integrals $\int_{M}\left\|H_{i}\right\|^{2}, i=1,2$, are finite. If the pages are taut, that is $H_{1}=0$, then $2 \sigma_{2}=-\left\|A_{1}\right\|^{2} \leq 0$ and the Ricci curvature of $M$ cannot be positive everywhere (if only the flow orthogonal to the pages has finite total curvature). Similarly, if the pages are umbilical, then $\sigma_{2} \geq 0$ and the Ricci curvature of $M$ cannot be negative everywhere (again, if the integrals mentioned above are finite).

Also, formula (25) for distributions arising from an OBD reduces significantly and provides results analogous to those mentioned above for open book decompositions with, say, pages of constant mean curvature and constant normal curvature.

Note that, open book decompositions can be defined and studied on manifolds of higher dimension. In general, the existence of them depends on the topology of manifolds under consideration (see, for example [20]), however, Lawson [10] proved that all odd-dimensional closed manifolds of dimension $>6$ admit OBDs. Certainly, our formulae can be applied to OBDs in higher dimensions as well.

Open Access This article is distributed under the terms of the Creative Commons Attribution 4.0 International License (http://creativecommons.org/licenses/by/4.0/), which permits unrestricted use, distribution, and reproduction in any medium, provided you give appropriate credit to the original author(s) and the source, provide a link to the Creative Commons license, and indicate if changes were made. 


\section{References}

1. Alexander, J.W.: Note on Riemann spaces. Bull. Am. Math. Soc. 26, 370-372 (1920)

2. Andrzejewski, K.: The Newton Transformation and Extrinsic Curvatures of Foliations and Distributions, Thesis. IMPAN, Warsaw (2010)

3. Andrzejewski, K., Walczak, P.: The Newton transformation and new integral formulae for foliated manifolds. Ann. Global Anal. Geom. 37(2), 103-111 (2010)

4. Asimow, D.: Average Gaussian curvature of leaves of foliations. Bull. Am. Math. Soc. 84, 131-133 (1978)

5. Baird, P., Wood, J.C.: Harmonic morphisms between Riemannian manifolds, London Math. Society Monographs (N.S.), vol. 29. Oxford University Press, Oxford (2003)

6. Brito, F., Langevin, R., Rosenberg, H.: Intégrales de courbure sur des variétés feuilletées. J. Differ. Geom. 16, 19-50 (1981)

7. Brito, F., Naveira, A.M.: Total extrinsic curvature of certain distributions on closed spaces of constant curvature. Ann. Global Anal. Geom 18, 371-383 (2000)

8. Brito, F., Walczak, P.: On the energy of unit vector fields with isolated singularities. Ann. Pol. Math. 73, 269-273 (2000)

9. Langevin, R., Walczak, P.: Conformal geometry of foliations. Geom. Dedicata 132, 135-178 (2008)

10. Lawson, T.: Open book decompositions for odd dimensional manifolds. Topology 17, 189-192 (1978)

11. Ranjan, A.: Structural equations and an integral formula for foliated manifolds. Geom. Dedicata 20, 85-91 (1986)

12. Reeb, G.: Sur la courboure moyenne des variétés intégrales d'une équation de Pfaff $\omega=0$. C. R. Acad. Sci. Paris 231, 101-102 (1950)

13. Reinhart, B.L.: Foliated manifolds with bundle-like metrics. Ann. Math. 69, 119-132 (1959)

14. Rovenski, V.: The partial Ricci flow for foliations. In: Rovenski, V., Walczak, P. (eds.) Geometry and Its Applications, pp. 125-155. Springer, Berlin (2014)

15. Rovenski, V., Walczak, P.: Integral formulae for foliated symmetric spaces. Math. Ann. 352, 223-237 (2012)

16. Rovenski, V., Walczak, P.: Topics in Extrinsic Geometry of Codimension-One Foliations. Springer Briefs in Mathematics. Springer, Berlin (2011)

17. Rovenski, V., Wolak, R.: Deforming metrics of foliations. Central Eur. J. Math. 11, 1039-1055 (2013)

18. Svensson, M.: Holomorphic foliations, harmonic morphisms and the Walczak formula. J. Lond. Math. Soc. 68, 781-794 (2003)

19. Walczak, P.: An integral formula for Riemannian manifold with two orthogonal complementary distributions. Coll. Math. 58, 243-252 (1990)

20. Winkelkemper, H.E.: Manifolds as open books. Bull. Am. Math. Soc. 79, 45-51 (1973) 